\title{
Measurement of six degree bi-directional motion error of linear stage
}

\author{
Ryoshu Furutani \\ Tokyo Denki University, School of Engineering, Senju-Asahi 5, Adachi, 120-8551 Tokyo, Japan
}

\begin{abstract}
We proposed the measurement system of the six degree of motion errors which is based on distance measurement by the laser interferometer. The system has six parallel laser beams and six ball lenses as the retroreflectors on the linear stage, which reflect the corresponding laser beams. In the proposed system, the error of axial direction is measured with the ordinary distance measurement method by laser interferometer. The vertical errors to the axial direction and the roll errors around the optical axis are measured by tilted beams using the wedge prism. The pitch and yaw errors in the vertical plane to the optical axis are measured by the difference between distance of two ball lenses. The former system can measure the displacement and the error angle in one-direction. The propose system are expanded and bi-directional displacement and error angle can be measured. In this paper, it is shown how to expand the measurement system. As a result, the maximum displacement errors in $\mathrm{x}, \mathrm{y}$ and $\mathrm{z}$ directions are $242 \mathrm{~nm}, 179 \mathrm{~nm}$ and $90 \mathrm{~nm}$. The maximum rotational errors around $\mathrm{x}, \mathrm{y}, \mathrm{z}$ axes are $1.75 \operatorname{arcsec}, 2.35 \operatorname{arcsec}$ and 1.67 arcsec.
\end{abstract}

\section{Introduction}

Six Degree-of-freedom (6DOF) motion errors of a linear stage of a machine tool, a coordinate measuring machine and so on should be measured simultaneously in order to improve their accuracies. As a result, the more accurate position and orientation can be obtained and higher compensation can be expected. However, it is difficult to measure 6DOF motion errors simultaneously because the stages have no enough space to setup conventional measuring instruments such as displacement sensors and autocollimators. Therefore, it is expected to develop the measuring instrument that can simultaneously measure the 6DOF motion errors. For these reasons, many researchers have developed the measuring method of the 6DOF motion errors [1-5] and the measuring system of 6 DOF motion errors that employs the principle of the Michelson interferometer to detect all direction displacement and rotational angle errors is developed because of the advantage of constructing high accurate measuring system with comparative ease [6-7]. However, the system proposed in [6-7] can measure 6 DOF motion errors in only one direction. In this paper, the system can be expanded to measure the 6 DOF motion errors in bidirection.

\section{Measurement Principle}

\subsection{Optical System}

The schematic diagram of the system is shown in Figure 1. The system is based on the length measurement by
Michelson interferometer. The moving plate (MP) which consisted of six target mirrors (TM) and two plates, was mounted on a stage as a measuring object in situ situation. The six ball lenses were adapted as the target mirrors. The refractive indices of those are nearly 2 . To fulfil retro reflection of a laser beam, the ball lenses were coated with silver film in a hemispheric part as shown in Figure 2. The other optical devices were fixed on an optical table. The laser beam emitted from laser source was expanded by two plano-convex lenses. The expanded laser beam was separated into the two laser beams by a beam splitter(BS). One of the two laser beams passed toward TMs and was partly tilted by three wedge prisms (WP). The other laser beam passed toward a reference mirror (RM). Each laser beam entered and was reflected by the target mirrors and the RM. Finally, the laser beam reflected from the target mirrors interferes with the laser beam reflected by the RM. When the MP moved, optical path difference between the two laser beams was varied. The displacements of the 6DOF motion errors of MP could be measured by counting the number of the interference fringes due to the two laser beams. The number of the interference fringes could be obtained by photo diodes (PD).

\subsection{Displacement measurement in $\mathrm{x}$ direction}

The schematic diagram of the principle of displacement measurement in $\mathrm{x}$ direction is shown in Figure 3. When MP moves to the $\mathrm{x}$ direction with a value of $\Delta \mathrm{x}$, the relation of the displacement $\Delta \mathrm{x}$ and the number of the interference fringes which are caused by the interference 


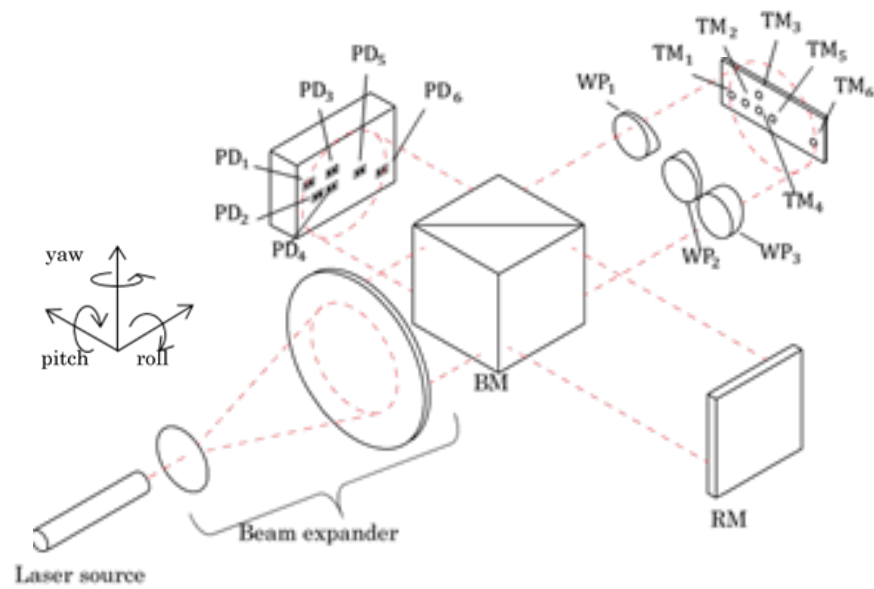

Figure 1. Configuration of optical system of 6 DOF laser interferometer.

of the laser beams reflected from the TM4 and the RM is shown as following equation (1).

$$
\Delta x=\frac{\lambda}{2} n_{4}
$$

Here, $\lambda$ denotes a wave length of the laser beam, $n_{4}$ denotes the number of interference fringe obtained by the $\mathrm{PD}_{4}$ which is irradiated by the laser beam reflected from $\mathrm{TM}_{4}$.

\subsection{Displacement measurement in y direction}

The schematic diagram of the principle of $y$ direction displacement measurement is shown in Figure 4. A part of the laser beam is tilted by the $\mathrm{WP}_{2}$ with the angle $\theta$ to the $\mathrm{x}$ axis in the $\mathrm{x}-\mathrm{y}$ coordinate system. When MP moves in the $y$ direction with the value of $\Delta y$, the optical path length of the tilted laser beam changes only $(\lambda / 2) n_{5}$ where $n_{5}$ is regarded as the number of interference fringes obtained by the $\mathrm{PD}_{5}$ which is irradiated by the laser beam reflected from TM5. The relation of the displacement $\Delta y$ and the number of the interference fringes which is caused by the interference of laser beams reflected from the $\mathrm{TM}_{5}$ and the RM is shown as following equation (2).

$$
\Delta y=\frac{\lambda}{2 \sin \theta} n_{5}
$$

\subsection{Displacement measurement in $z$ direction and roll angle measurement around $x$ axis}

The schematic diagram of the principle of $\mathrm{z}$ direction and roll angle measurement is shown in Figure 5 and Figure 6. $\mathrm{TM}_{1}, \mathrm{TM}_{6}, \mathrm{WP}_{1}$ and $\mathrm{WP}_{3}$ are used in the measurement. The displacement in $\mathrm{z}$ direction can be measured by the same method as that in y direction. The displacement in $\mathrm{z}$ direction is measured by the both laser beams tilted by the both WP1 and WP3 of which primary axes are rotated with 90 degree around $\mathrm{x}$ axis. Thus, the displacement $\Delta \mathrm{z}$ and the roll angle $\Delta \theta_{\mathrm{x}}$ can be measured as the following equation (3) and equation (4).

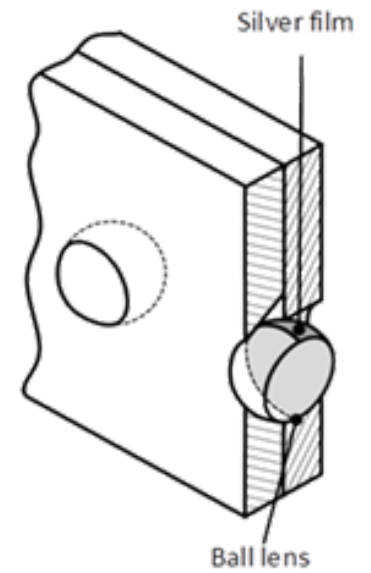

Figure 2. Moving plate(MP).

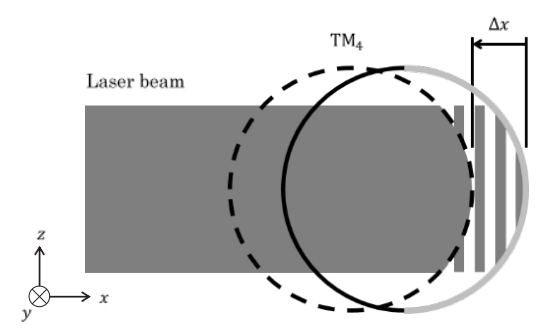

Figure 3. Measurement principle of x-displacement.

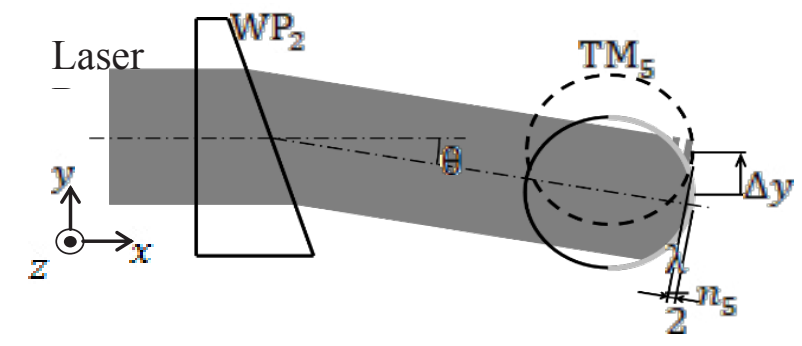

Figure 4. Measurement principle of y-displacement

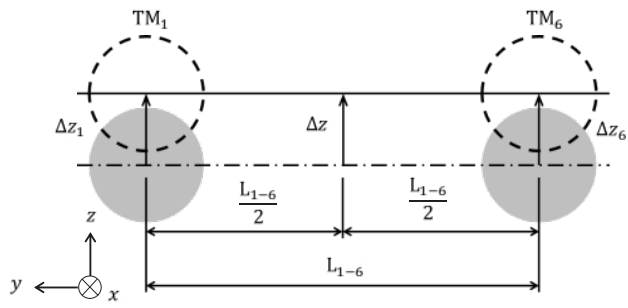

Figure 5. Measurement principle of z-displacement.

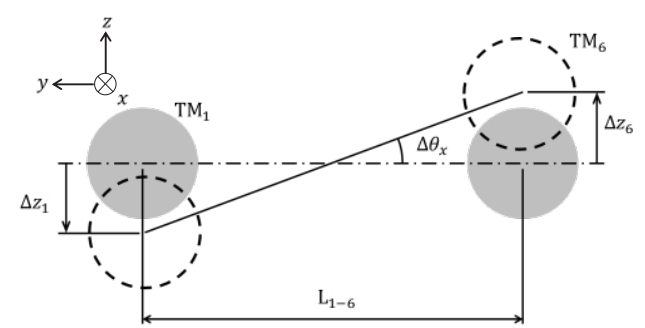

Figure 6. Measurement principle of roll angle around x-aixs. 


$$
\begin{gathered}
\Delta z=\frac{\lambda}{4 \sin \theta}\left(n_{1}+n_{4}\right) \\
\Delta \theta_{x}=\tan ^{-1}\left(\frac{\lambda}{2 L_{1-6} \sin \theta}\left(n_{1}-n_{6}\right)\right)
\end{gathered}
$$

Here, $\mathrm{L}_{1-6}$ denotes the distance between $\mathrm{TM}_{1}$ and $\mathrm{TM}_{6}$.

\subsection{Pitch and yaw angle measurement in the vertical plane to $x$ axis}

The schematic diagram of the principle of pitch angle measurement is shown in Figure 7. The pitch angle can be calculated by $\mathrm{x}$ direction displacement of $\mathrm{TM}_{3}$ and $\mathrm{TM}_{4}$ and the distance between $\mathrm{TM}_{3}$ and $\mathrm{TM}_{4}$. The $\mathrm{x}$ direction displacement of $\mathrm{TM}_{3}$ and $\mathrm{TM}_{4}$ can be measured by the same method of $\Delta \mathrm{x}$ mentioned in 2.2. The yaw angle can be measured as similar as the pitch angle using $\mathrm{TM}_{2}$ ad $\mathrm{TM}_{4}$ as shown in Figure 8. Thus, the pitch and yaw angle $\Delta \theta_{\mathrm{y}}$ and $\Delta \theta_{\mathrm{z}}$ are expressed as following equation (5) and equation (6).

$$
\begin{aligned}
& \Delta \theta_{y}=\tan ^{-1}\left(\frac{\lambda}{2 L_{3-4}}\left(n_{3}-n_{4}\right)\right) \\
& \Delta \theta_{z}=\tan ^{-1}\left(\frac{\lambda}{2 L_{2-4}}\left(n_{2}-n_{4}\right)\right)
\end{aligned}
$$

- Here, $\mathrm{L}_{3-4}$ denotes the distance between $\mathrm{TM}_{3}$ and $\mathrm{TM}_{4}, \mathrm{~L}_{2-4}$ denotes the distance between $\mathrm{TM}_{2}$ and $\mathrm{TM}_{4}, \mathrm{n}_{2}, \mathrm{n}_{3}$ and $\mathrm{n}_{4}$ denotes the number of interference fringes obtained by the PDs which are irradiated by the laser beam reflected from $\mathrm{TM}_{2}, \mathrm{TM}_{3}$ and $\mathrm{TM}_{4}$.

\subsection{Measurement in bi-directional way}

In the method mentioned above, the moving direction could not be distinguished. The photo diodes are replaced with the bi-photo diodes. By using the bi-photo diodes, the moving direction is able to be distinguished as shown in Figure 9.

\section{Experiments}

\subsection{Experimental devices}

The specifications of the optical devices are shown in Table 1. The MP was mounted on the xyz- and rotational stages, and driven by these stages. The displacement of the MP was measured 5530 Laser Calibration System (Agilent Technologies) as reference. Three axes piezo nanometer positioning stage (Physik Intrumente, P733.3DD) and three rotation stages(Suruga Seiki, KGW06100, KGW06075 and KRW06360) were used. The specifications of the used positioning stages are shown in Table 2.

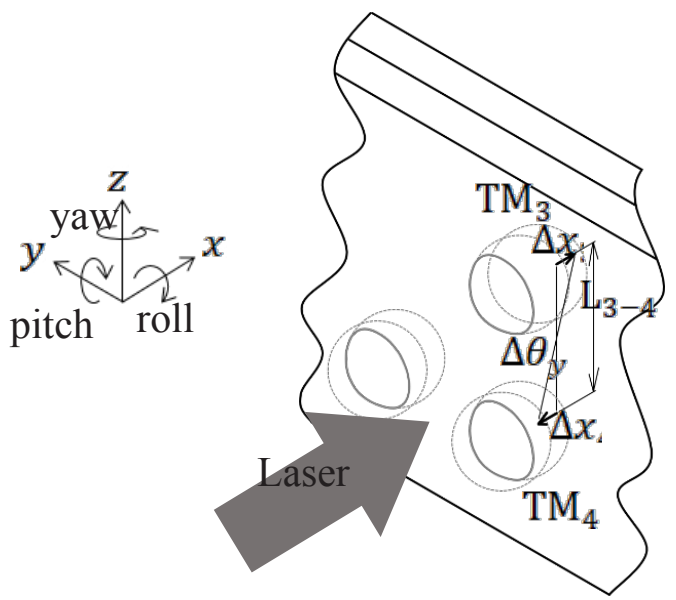

Figure 7. Measurement principle of pitch angle in the plane vertical to the optical axis.

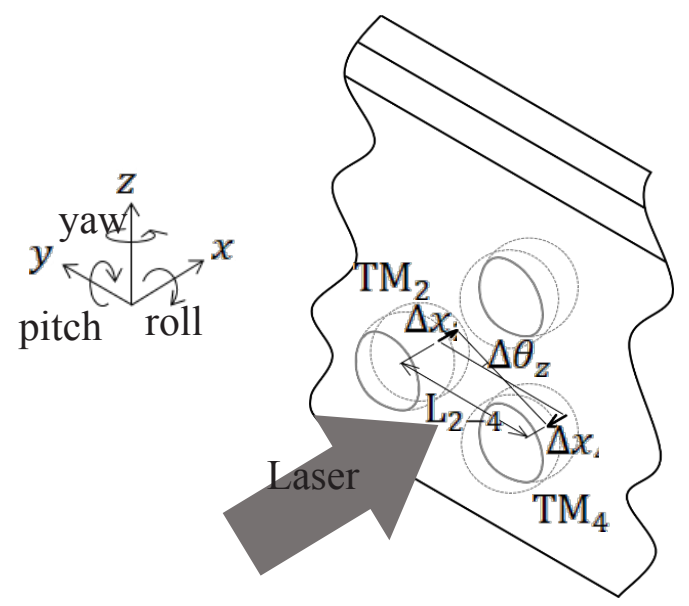

Figure 8. Measurement principle of yaw angle in the plane vertical to the optical axis.
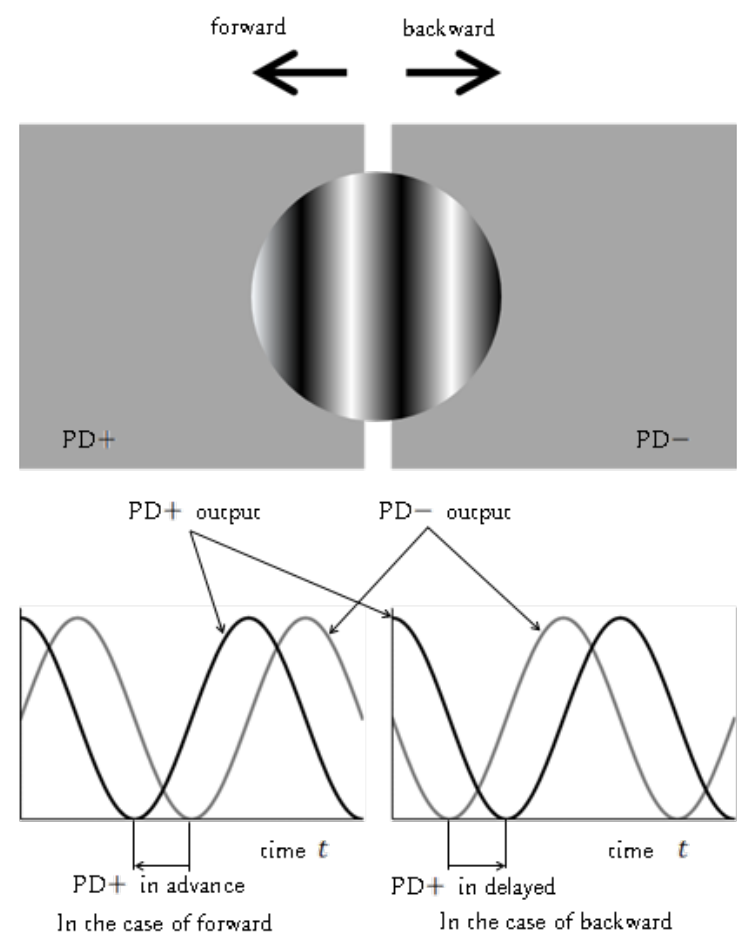

Figure 9. Distinguish of forward and backward direction. 
Table 1. Specification of the optical system.

\begin{tabular}{|c|c|}
\hline Item & value \\
\hline$\lambda:$ Wave length of the laser beam & $632.57 \mathrm{~nm}$ \\
\hline$\theta:$ Tilted angle of the laser beam & 10 degree \\
\hline Refractive indices of the ball lens & 2.003 \\
\hline $\mathrm{L}_{1-6}:$ Distance between $\mathrm{TM}_{1}$ and $\mathrm{TM}_{6}$ & $48.06 \mathrm{~mm}$ \\
\hline $\mathrm{L}_{3-4}:$ Distance between $\mathrm{TM}_{3}$ and $\mathrm{TM}_{4}$ & $7.92 \mathrm{~mm}$ \\
\hline $\mathrm{L}_{2-4}:$ Distance between $\mathrm{TM}_{2}$ and $\mathrm{TM}_{4}$ & $8.19 \mathrm{~mm}$ \\
\hline
\end{tabular}

Table 2. Specification of stages.

\begin{tabular}{|c|c|c|c|}
\hline Margin & Direction & Resolution & Repeatability \\
\hline $\begin{array}{c}\text { XYZ-Stage } \\
{[\mathrm{P}-733.3 \mathrm{DD}]}\end{array}$ & $\mathrm{x}, \mathrm{y}$ and $\mathrm{z}$ & $0.1 \mathrm{~nm}$ & $\begin{array}{c}\text { 2nm, 2nm and } \\
1 \mathrm{~nm}\end{array}$ \\
\hline $\begin{array}{c}\text { Gonio-Stage } \\
{[\text { KGW06100] }}\end{array}$ & Roll & $\begin{array}{c}0.002466 \\
\text { degree }\end{array}$ & 0.003 degree \\
\hline $\begin{array}{c}\text { Gonio-Stage } \\
{[\text { KGW06075] }}\end{array}$ & Pitch & $\begin{array}{c}0.0032 \\
\text { degree }\end{array}$ & 0.003 degree \\
\hline $\begin{array}{c}\theta \text {-Stage } \\
\text { KRW0636] }]\end{array}$ & Yaw & 0.004 degree & 0.01 degree \\
\hline
\end{tabular}

\subsection{Experiment of detection of Bi-directional movement}

In order to examine whether the bi-photo diodes can detect the moving direction, when the MP moves $5 \mu \mathrm{m}$ along z-axis. The result is shown in Figure 10. Figure 10 a) shows a result when the MP moves forward along $\mathrm{z}-$ direction. Figure 10. b) shows a result when the MP moves backward along z-direction. It is proved that the moving directions are detected by comparing the phase of output signal of PD1+ and output signal of PD1-.

\subsection{Experiment of movements}

In this system, the principle of displacement measurement of $y$ and $z$ axis are similar, the principle of angle measurement of pitch and yaw are also similar. Therefore, the displacement in $\mathrm{x}$ and $\mathrm{y}$ axes and the angle roll and pitch are measured and compared with 5530 Laser Calibration System. Every measurement is iterated 5 times.

Figure 11. shows the result of displace measurement in $\mathrm{x}$ - and $\mathrm{y}$ - axis.

Figure 12. shows the result of angle measurement in roll and pitch.

In these measurements, the maximum displacement errors in $\mathrm{x}, \mathrm{y}$ and $\mathrm{z}$ directions are $242 \mathrm{~nm}, 179 \mathrm{~nm}$ and $90 \mathrm{~nm}$. The maximum rotational errors (roll, pitch and yaw) are $1.75 \operatorname{arcsec}, 2.35 \operatorname{arcsec}$ and 1.67 arcsec. the results of this system meet with the reference instrument.

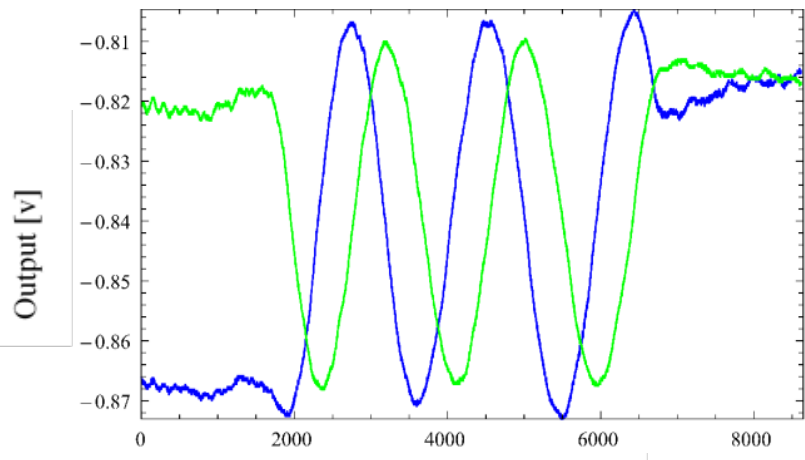

Time [ms]
a) The output signal of PD1 in forward movement.

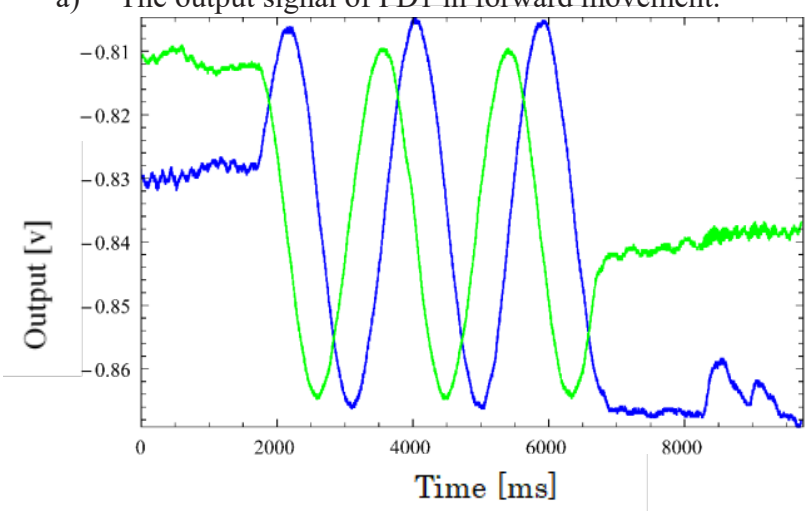

b) The output signal of I 11 im vaunwaru movement.

Figure 10. The output signal of PD1+(blue line) and PD(green line) when the MP moves $5 \mu \mathrm{m}$ in z-direction.

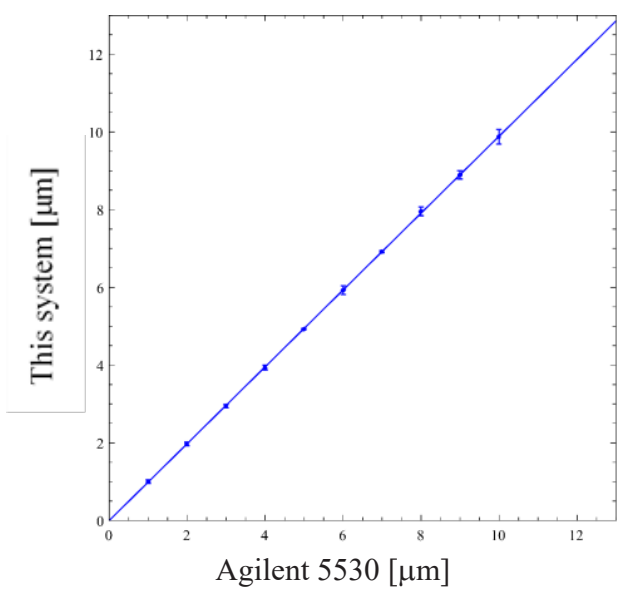

a) $\mathrm{x}$-direction movement.

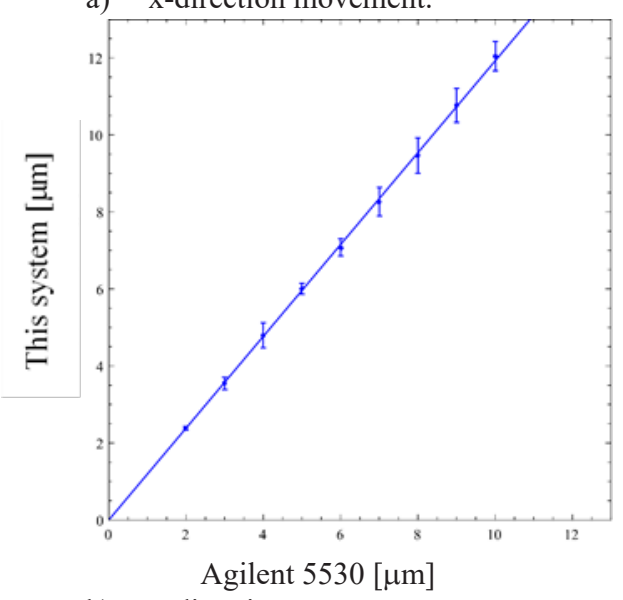

b) y-direction movement.

Figure 11. The relationship between the displacements of this system and Agilent 5530. 


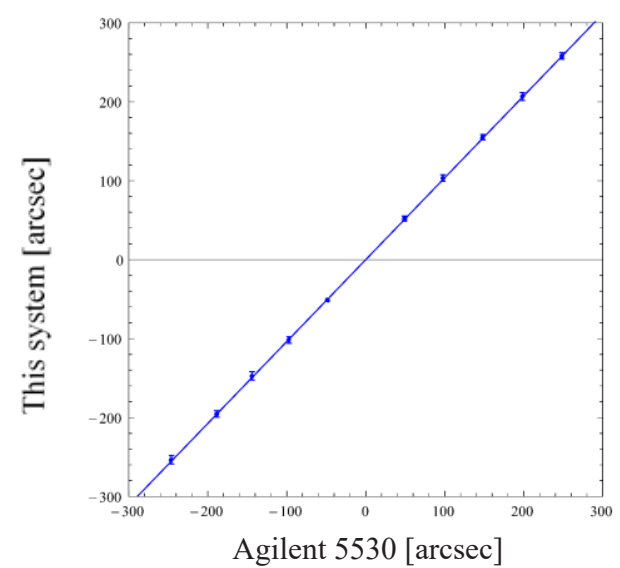

b) Roll angle movement.

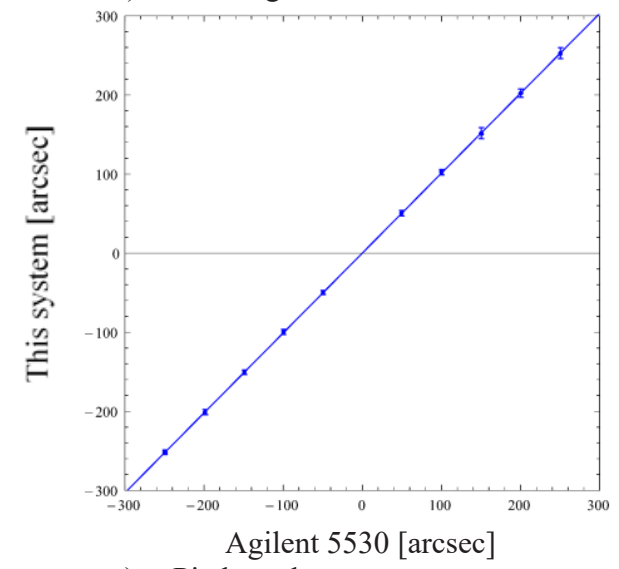

c) Pitch angle movement.

Figure 12. The relationship between the angle movement of this system and Agilent 5530.

\section{Conclusion}

The measurement system which can measure 6DOF of the stage was proposed. The proposed optical system was composed and the measurement result of this system were compared with 5530 Laser calibration system as reference. They are enough good to meet.

However, these experiments were performed independently. One displacement and/or one angle movement must affect the other displacements and angles. So two or more movements are composed and should be measured simultaneously in future work.

\section{References}

1. S. Shimizu, H. S. Lee and N. Imai, Int.J.Japan Soc. Prec. Eng. 28, 273 (1994)

2. C.H.Liu, W.Y.Jywe, C.C.Hsu, Review of Scientific Instrument 76,055110 (2005)

3. Q.Feng, B.Zhang, C.Cui, C.Kuang, Y.Zhai, F.You, Optics Express 22,21,25805(2008)

4. K.C.Fan, M.J.Chen,W.M.Huang,Int.J.Machine Tools and Manufacture 38, 155 (1998)

5. K.C.Fan, M.J.Chen, Precision Engineering 24, 15 (2000)

6. J.Izawa, S.Kanno, M.Ozaki, R.Furutani, Proc. ISMTII 2015 (2015)

7. R.Furutani, Materials Science and Engineering 211 b012001 (2017) 